\title{
Cognitive impairment patterns in schizophrenia and affective disorder
}

\author{
MICHAEL ALAN TAYLOR, RICHARD ABRAMS \\ From the Department of Psychiatry and Behavioural Sciences, University of Health Sciences, The Chicago \\ Medical School, North Chicago, Illinois, USA
}

SUMmarY A battery of neuropsychological tasks was used to study 62 schizophrenics, 67 melancholics, and 30 manics satisfying specific research diagnostic criteria, and 42 normal subjects. Two patterns of neuropsychological impairment among patients were identified by factor analysis. The first pattern of bifrontal, non-dominant hemisphere dysfunction was shared by schizophrenics and affectively ill patients, whereas the second pattern of dominant temporo-parietal-occipital impairment was most frequent in a subgroup of schizophrenics. These relationships remained after accounting for the effects of age, gender, handedness and drugs received at time of testing.

A prevalent hypothesis of cerebral hemispheric dysfunction in the major psychoses posits that schizophrenic patients evidence cognitive impairment of the dominant hemisphere, whereas affectively ill individuals are impaired in the non-dominant hemisphere. ${ }^{12}$ Data from neuropsychological, ${ }^{134}$ electrophysiological, ${ }^{125}$ and brain imaging ${ }^{67}$ studies are cited in support of this view. Our own studies, ${ }^{8-10}$ also suggest that, compared with controls, affectively ill individuals demonstrate primarily non-dominant hemisphere impairment, but that schizophrenics have bilateral impairment which is relatively worse in the dominant frontotemporal regions.

If hemispheric dysfunction in the major psychoses is to be related to the pathophysiology of these disorders, clarification of the patterns of dysfunction across diagnostic categories is a necessary step. The present study attempts to characterise these patterns of impairment by comparing cognitive functioning in a sample of rigorously diagnosed schizophrenic and affectively ill patients with that of a sample of normal controls.

\section{Method}

This investigation was carried out over a 3 year period ending in June 1980. We included for study all patients admitted to an acute treatment, university-affiliated psychiatric inpatient service of a public hospital, who had no

Address for reprint requests: Dr MA Taylor. Chicago Medical School, 3333 Green Bay Rd, North Chicago, Ill 60064, USA.

Received 18 July 1986 and in revised form 3 November 1986. Accepted 6 November 1986 motor disability, a corrected visual acuity of at least 20/30 in each eye, were able to complete 2.5 hours of neuropsychological testing, and who satisfied the following research criteria:

\section{For schizophrenia (all required):}

1. No prominent affective symptoms.

2. No evidence of coarse brain disease or medical condition known to produce schizophrenic-like symptoms.

3. Clear consciousness.

4. At least one of the following: emotional blunting, at least one first-rank symptom, or formal thought disorder.

These criteria have been shown to predict most accurately the latent class or core diagnostic concept of schizophrenic. ${ }^{11}$

\section{For melancholia (all required):}

1. Sad, dysphoric, or anxious mood.

2. At least three of (a) to (f):
(a) early morning waking
(b) diurnal mood swing (worse in am)
(c) $>2.2 \mathrm{~kg}$ weight loss in three weeks
(d) retardation or agitation
(e) suicidal thoughts/behaviour
(f) feelings of guilt/hopelessness/worthlessness

3. No diagnosable coarse brain disease, no use of steroids or reserpine in past month, no medical illness known to cause depressive symptoms.

Each patient who satisfied these criteria also satisfied DSMIII criteria for major depressive disorder, melancholic subtype.

For mania (all required):

1. No evidence of coarse brain disease, psychostimulant drug abuse in the month prior to episode onset, or medical illness known to cause manic symptoms.

2. Hyperactivity during index episode. 
3. Rapid or pressured speech.

4. Euphoric, expansive or irritable mood with broad affect.

These criteria have been shown to predict most accurately the latent class or core diagnostic concept of mania. ${ }^{12}$

At a later date we tested a sample of normal controls who had no motor disability, visual acuity of at least 20/30 in each eye with corrective lenses and no history of psychiatric or neurological disorder, alcoholism, drug abuse, or systemic illness known to affect brain function. Each patient and control gave informed consent.

Each subject received a battery of neuropsychological tasks administered by a trained technician who was unaware of the patients' research diagnosis. We selected cognitive tasks of known reliability which had been successfully used in studies of psychiatric patients and which had been found valid in predicting generalised as well as regional higher cortical function. Testing required about $2 \frac{1}{2}$ hours and included the following:

I. Evaluation of soft neurological signs (for example, motor overflow, double simultaneous discrimination, grasp reflex, motor impersistence, adventitious motor overflow, snout and grasp reflexes, gegenhalten, post hyperventilation apnoea, echophenomena, motor perseveration). ${ }^{1314}$

II. Aphasia Screening Test. ${ }^{1516}$

III. "Minimental State". 1718

IV. Halstead-Reitan Battery (selected items: finger tapping, tactile form recognition). ${ }^{19-21}$

V. Luria Nebraska Battery ${ }^{2-24}$ (selected items: motor function tasks, visual perception and spatial orientation items, phonemic hearing, expressive speech items, naming, reading comprehension, arithmetical operations, verbal and visual memory, logical relationships, discursive intellect items, rhythm reproduction, cutaneous and kinaesthetic functions, spatial orientation)

VI. Tachistoscopic stimulation (for example, letters and nonsense figures to visual hemi-fields). ${ }^{25-29}$ We considered a deficit to be present if a subject made $20 \%$ or more errors to any visual field. We considered the right hemisphere dysfunctional if percent left visual field figure errors was greater than percent right visual field figure errors; whereas we considered the left hemisphere dysfunctional if percent right visual field letter errors was greater than percent left visual field letter errors.

Many of these tasks have known reliability and validity in neurological populations and were administered according to standardised procedures referenced above.

Handedness was determined by requiring the subject to demonstrate the use of common objects with his preferred hand (for example, throwing a ball, using scissors) and by timed signature writing with each hand, the faster hand by at least 5 seconds considered preferred. Each patient was classified blind to diagnosis as purely dextral, mixed, or purely sinistral. In the first two groups, the dominant hemisphere was assumed to be the left, whereas for pure sinistrals, the dominant hemisphere was assumed to be the right.

Upon completion of data collection, all identifying information was removed from each protocol, all patient and control protocols randomly mixed, and each rated "blindly" for hemispheric, regional cortical and global impairment as follows: impairment for each hemispheric region (dominant and nondominant frontal, temporal, parietal, and occipital) and global impairment: 1-none, 2-mild, 3-moderate, 4-marked, 5-severe. For laterality of impairment: 1-none, 2-dominant greater than nondominant, 3-symmetrical, 4-nondominant greater than dominant, 5-nondominant only. Thus, each protocol received 10 separate scores. Impairment localisation was determined by standard clinical neuropsychological methods $s^{149-24262729}$ and adhered to established relationships between cognitive function and regional cortical structure. ${ }^{30-32}$

\section{Results}

Sixty-two schizophrenics, 67 melancholics, 30 manics and 42 normal subjects completed the study. Table 1 displays demographic and some clinical comparisons for the four groups. Although the three patient groups were significantly older than controls $(F=$ $16.99, \mathrm{df}=3, \mathrm{p}<0.001)$, the patient groups were not significantly different in age from each other $(F=$ $2 \cdot 15, \mathrm{df}=2, \mathrm{p}=0 \cdot 12)$. There were significantly more women in the control than patient groups $\left(\chi^{2}=\right.$ 44.25 , df $=3, p=0.001$ ), and among the patient groups there were significantly more women in the manic than in the other two diagnostic groups $\left(\chi^{2}=\right.$ $9 \cdot 12, \mathrm{df}=2, \mathrm{p}=0.01)$. There were also differences in

Table 1 Demographic and clinical comparisons between patient groups and normal controls

\begin{tabular}{|c|c|c|c|c|}
\hline Variable & $\begin{array}{l}\text { Schizophrenics } \\
(n=62)\end{array}$ & $\begin{array}{l}\text { Melancholics } \\
(n=67)\end{array}$ & $\begin{array}{l}\text { Manics } \\
(n=30)\end{array}$ & $\begin{array}{l}\text { Controls } \\
(n=42)\end{array}$ \\
\hline $\begin{array}{l}\text { Mean index age and (SD) } \\
\% \text { Male } \\
\% \text { Pure Sinistral } \\
\% \text { Mixed handedness }\end{array}$ & $\begin{array}{l}43 \cdot 9(11 \cdot 5) \\
90 \cdot 3 \\
6 \cdot 6 \\
13 \cdot 1\end{array}$ & $\begin{array}{l}48 \cdot 1(12 \cdot 0) \\
94 \cdot 0 \\
1 \cdot 5 \\
10 \cdot 6\end{array}$ & $\begin{array}{l}47 \cdot 1(12 \cdot 2) \\
73 \cdot 3 \\
10 \cdot 0 \\
23 \cdot 3\end{array}$ & $\begin{array}{l}32 \cdot 5(10 \cdot 6) \\
45 \cdot 2 \\
2 \cdot 4 \\
23 \cdot 8\end{array}$ \\
\hline $\begin{array}{l}\text { Treatment at time of testing*: } \\
\text { Neuropletic only } \\
\text { Lithium only } \\
\text { Combination Li and neuroleptic } \\
\text { Antidepressant only } \\
\text { Sedative only } \\
\text { None }\end{array}$ & $\begin{array}{r}17 \\
0 \\
1 \\
1 \\
7 \\
33\end{array}$ & $\begin{array}{r}2 \\
1 \\
1 \\
2 \\
17 \\
40\end{array}$ & $\begin{array}{r}2 \\
9 \\
14 \\
0 \\
1 \\
1\end{array}$ & $\begin{array}{r}0 \\
0 \\
0 \\
0 \\
0 \\
42\end{array}$ \\
\hline
\end{tabular}

*Treatment data missing from some subjects. 
Rotated loadings

$\begin{array}{ll}\text { L front } & 0.883 \\ \text { R tront } & 0.884 \\ \text { L temp } & 0.056 \\ \text { R temp } & 0.377 \\ \text { L par } & 0.161 \\ \text { R par } & 0.796 \\ \text { L occip } & 0.100 \\ \text { R occip } & 0.695\end{array}$

Fig Factor 1 (left), factor 2 (right)

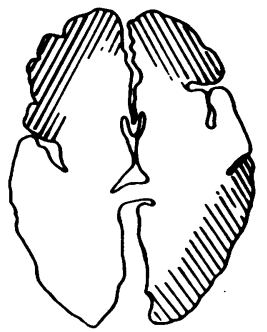

treatments received among the patient groups, with most depressives being unmedicated at time of testing, while most schizophrenics were receiving neuroleptics and most manics were receiving lithium or a combination of lithium and neuroleptic.

We performed a principal components factor analysis based on the regional cortical impairment scores of patients and controls. One-hundred and ninetyeight protocols were included. Eight factors were generated, only two of which had eigenvalues greater than 1.0. Factor 1 (eigenvalue $=4 \cdot 00$ ) accounted for 1.63 ) accounted for $20.41 \%$ of the variance. None of the other six factors accounted for more than $10 \%$ of the variance. The figure displays diagrams of the two factors and their loading scores after varimax rotation for each cortical region. Factor loading less than 0.5 are not displayed. The diagrams show that factor dominant) hemisphere impairment, and factor 2 to a pattern of left (dominant) hemisphere temporoparieto-occipital impairment.

We assessed the relationship between factor 1 and 2 and group assignment by analyses of variance and independent $t$ tests. Table 2 displays the mean factor scores for the patient groups and controls. The four groups differ significantly on factor 1 , primarily due to controls having a negative mean factor 1 score (better cognitive performance) $(\mathrm{F}=23.07, \mathrm{df}=3, \mathrm{p}$ $=0.0001$ ). Among the three patient groups, manics and depressives are not significantly different from each other $(F=2.47$, df $=1, p=0.12)$, whereas schizophrenics have a significantly higher mean factor $49.95 \%$ of the variance, while factor 2 (eigenvalue 1 corresponds to a pattern of bifrontal, right (non-

Rotated loadings

$\begin{array}{ll}\text { L front } & 0.142 \\ \text { R front } & 0.024 \\ \text { L temp } & 0.882 \\ \text { R temp } & 0.491 \\ \text { L par } & 0.855 \\ \text { R par } & 0.231 \\ \text { L occip } & 0.830 \\ \text { R occip } & 0.137\end{array}$

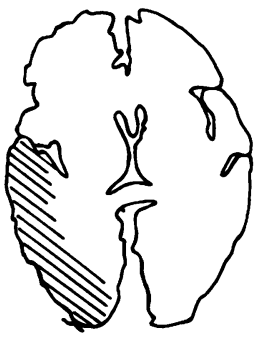

1 score (worse cognitive performance) than do depressives $(F=37 \cdot 36, d f=1, p=0 \cdot 0001)$. Schizophrenics and a combined affective disorder group ( $N$ $=96$, mean $(\mathrm{SD})=0.154(1.011))$ do not significantly differ on mean factor 1 scores $(t=1 \cdot 64$, $\mathrm{df}=154, \mathrm{p}=0 \cdot 10)$. The four groups also differ significantly on factor 2 , with affectives and controls having negative mean factor scores (better cognitive performance), and schizophrenics having a high positive mean factor 2 score (worse cognitive performance) $(\mathrm{F}=6.87, \mathrm{df}=3, \mathrm{p}=0.0002)$. Schizophrenics also differ significantly $(t=2 \cdot 91, \mathrm{df}=154$, $\mathrm{p}=0.004)$ from a combined affective disorder group $(\mathrm{N}=96$, mean (SD) $=-0 \cdot 100(0.909))$. Thus, controls show virtually no pattern of impairment, affectives predominantly a bifrontal, right (nondominant) hemisphere impairment pattern (factor 2) and schizophrenics a bilateral impairment pattern (factor 1 and 2).

A correlation analysis revealed age $(r=0.26$, $p=0.001)$, gender $(r=0.17, p=0.022)$ and drugs administered at time of testing $(r=0.21, p=0.006)$ to correlate with factor 1 , and gender $(r=0.21$,

Table 2 Mean (SD) factor scores for patients and controls

\begin{tabular}{lcc}
\hline Group & $\begin{array}{l}\text { Factor } 1 \\
\text { mean }(S D)\end{array}$ & $\begin{array}{l}\text { Factor 2 } \\
\text { mean }(S D)\end{array}$ \\
\hline Schizophrenia $(\mathrm{n}=60)$ & $0.410(0.839)$ & $0.415(1.299)$ \\
Mania $(\mathrm{n}=29)$ & $0.398(1.067)$ & $-0.323(0.646)$ \\
Depression $(\mathrm{n}=67)$ & $0.048(0.976)$ & $-0.003(0.991)$ \\
Control $(\mathrm{n}=42)$ & $-0.938(0.469)$ & $-0.364(0.224)$ \\
\hline
\end{tabular}

Table 3 Hierarchial multiple regression analyses of the relationship between diagnosis and cognitive impairment factor

\begin{tabular}{|c|c|c|c|c|c|c|}
\hline Dependent variables & $\begin{array}{l}\text { Independent variables } \\
\text { entered }\end{array}$ & Multiple $R$ & $R^{2}$ & $R^{2}$ change & $F$ & Significance \\
\hline $\begin{array}{l}\text { Factor } 1 \\
\text { Factor } 2\end{array}$ & $\begin{array}{l}\text { Age, drugs, gender } \\
\text { Diagnosis } \\
\text { Gender } \\
\text { Diagnosis }\end{array}$ & $\begin{array}{l}0.364 \\
0.402 \\
0.214 \\
0.307\end{array}$ & $\begin{array}{l}0 \cdot 133 \\
0 \cdot 162 \\
0 \cdot 046 \\
0.094\end{array}$ & $\begin{array}{l}0.133 \\
0.138 \\
0.046 \\
0.048\end{array}$ & $\begin{array}{l}7 \cdot 12 \\
6 \cdot 78 \\
7 \cdot 36 \\
7 \cdot 94\end{array}$ & $\begin{array}{l}0.001 \\
0.028 \\
0.007 \\
0.005\end{array}$ \\
\hline
\end{tabular}


$p=0.006)$ to correlate with factor 2 . As these results and gender differences between patient groups potentially relate to cognitive function and therefore with the factor structure, we performed a hierarchial multiple regression analysis (SCSS forced-entry model) to account for the variance explained by these variables before we examined the relationship between each factor and the dichotomous variable of group assignment (schizophrenia or affective disorder). For each analysis, diagnosis was established as the dependent variable; the independent variables of age, gender and drug administration (coded present or absent) were entered and then factor 1 or 2 was entered. Table 3 displays this analysis which reveals that, after accounting for the variance of age, gender and drugs, there is a significant relationship between factor 1 and diagnosis, schizophrenics having a significantly greater correlation with factor 1 than do affectives (combined). After accounting for the variance of gender there is also a significant relationship between factor 2 and schizophrenia, but not between factor 2 and affectives.

As group means can be unduly influenced by outlyers, we inspected the histogram plots of factor 1 and 2 scores for patients and controls. We considered subjects with negative factor scores to be without impairment. For factor $1,100 \%$ of controls, $59 \%$ of affectives and $39 \%$ of schizophrenics had negative scores. For factor $2,93 \%$ of controls, $70 \%$ of affectives and $55 \%$ of schizophrenics had negative scores.

The median for positive scores of all subjects was 1.0. We considered this to be a reasonable threshold point for impairment and therefore interpreted a factor score of less than 1 to represent minimal impairment, and a factor score of greater than 1 to represent clinically important impairment. No control had a factor 1 or 2 score above 1 .

For factor 1 there were no significant betweengroup differences in the distribution of scores above and below the threshold of impairment (median). Table 4 displays the separation of factor 2 scores above and below the threshold of impairment point for the controls and patient groups. Controls are quite different from patients (all but three controls had negative factor 2 scores). The distribution among

Table 4 Distribution of Factor 2 scores above and below the threshold of impairment

\begin{tabular}{llc}
\hline & \multicolumn{2}{l}{ Factor 2 score } \\
\cline { 2 - 3 } Group & $<1 \cdot 0$ & $>1 \cdot 0$ \\
\hline Controls $(\mathrm{n}=42)$ & 42 & 0 \\
Manics $(\mathrm{n}=30)$ & 27 & 3 \\
Depressives $(\mathrm{n}=67)$ & 56 & 11 \\
Schizophrenics $(\mathrm{n}=62)$ & 42 & 20 \\
\hline
\end{tabular}

the three patient groups is also significantly different $\left(\chi^{2}=7.66, \mathrm{df}=2, \mathrm{p}=0.02\right)$ with only the rare manic having a factor 2 score suggestive of posterior dominant hemisphere impairment, compared with about $16 \%$ of depressives and over one-third of schizophrenics.

\section{Discussion}

We have identified two neuropsychological patterns of impairment among psychotic patients. One pattern of bifrontal, non-dominant hemisphere dysfunction (factor 1) although having a greater association with schizophrenics, is common to both schizophrenics and affectively ill patients. The second pattern of dominant temporo-parietal-occipital dysfunction (factor 2) is more specific to schizophrenia. The relationship between these patterns of dysfunction and diagnosis remains after accounting for the effects of age, gender and drugs received at time of testing.

The pattern of bifrontal, non-dominant hemisphere impairment in affective disorder is consistent with many other reports. ${ }^{133-36}$ The fact that schizophrenics also share this pattern of dysfunction suggests the pattern reflects either shared attentional deficits or shared underlying brain dysfunction, or that, although schizophrenics and affectives perform poorly on tasks related to non-dominant hemisphere function, their poor performances are due to different pathophysiologies. Further study is needed to resolve this issue.

The relationship between schizophrenia and dominant hemisphere impairment is not suprising, but the distribution of factor 2 scores among the schizophrenics suggests that important posterior dominant hemisphere impairment is limited to a subgroup of such patients. From studies of the speech and language impairment in schizophrenia, ${ }^{3738}$ it is possible that this may be a subgroup with formal thought disorder; future assessments of schizophrenics with and without formal thought disorder may aid in further characterising the nature of posterior dominant hemisphere impairment in schizophrenia.

The fact that some depressives also exhibit important dominant hemisphere impairment is intriguing in light of studies ${ }^{39}$ suggesting an association between the acute expression of sadness and left hemisphere lesions. Intuitively, one might predict that bipolar depressives would be similar to manics, with unipolar depressives exhibiting posterior dominant hemisphere impairment; this will require further study.

\section{References}

1 Flor-Henry P. Laterlized temporal-limbic dysfunction 
and psychopathology. Ann NY Acad Sci 1976;280: 777-96.

2 Gruzelier JH, Hammond N. Schizophrenia: a dominant hemisphere temporal limbic disorder. Res Comm Psychol Psychiatr Behav 1976;1:33-72.

3 Gur RE. Left hemisphere dysfunction and left hemisphere overactivation in schizophrenia. J Abnorm Psychol 1978;87:226-38.

4 Lishman WA, Toone BK, Colbourn CJ, McMeekan ERL, Mance RM. Dichotic listening in psychotic patients. Br J Psychiatry 1978;132:333-41.

5 Roemer RA, Shagass C, Straumanis JJ, Amadeo M. Pattern evoked potential measures suggesting lateralized hemispheric dysfunction in chronic schizophrenics. Biol Psychiatry 1978;13:185-202.

6 Gur RE, Gur RC, Skolnick BE, et al. Brain function in psychiatric disorders. III. Regional cerebral blood flow in unmedicated schizophrenics. Arch Gen Psychiatry 1985;43:329-34.

7 Morihisa JM, Duffy FH, Wyatt RJ. Brain electrical activity mapping (BEAM) in schizophrenic patients. Arch Gen Psychiatry 1983;40:719-28.

8 Taylor MA, Abrams R. Cognitive dysfunction in schizophrenia. Am J Psychiatry 1984;141:196-201.

9 Taylor MA, Abrams R. Cognitive dysfunction in mania. Comp Psychiat 1986;27:186-91.

10 Abrams R, Taylor MA. Cognitive dysfunction in melancholia. Psychol Med (in press).

11 Young MA, Tanner MA, Meltzer HY. Operational definitions of schizophrenia: what do they identify? $J$ Nerv Ment Dis 1982;170:443-7.

12 Young MA, Abrams R, Taylor MA, Meltzer HY. Establishing diagnostic criteria for mania. J Nerv Ment Dis 1983;171:676-82.

13 Quitkin F, Rifkin A, Klein DF. Neurologic soft signs in schizophrenia and character disorders. Arch Gen Psychiatry 1979;33:845-53.

14 Taylor MA, Abrams R, Faber R, Almy G. Cognitive tasks in the mental status examination. J Nerv Ment Dis 1980;168:167-70.

15 Heimburger RF, Reitan RM. Easily administered written test for lateralizing brain lesions. $J$ Neurosurg 1961;18:301-2.

16 Wheeler L, Reitan RM. Presence and laterality of brain damage predicted from responses to a short aphasia screening test. Percept Mot Skills 1962;15:783-99.

17 Folstein MF, Folstein SW, McHugh PR. "Mini-Mental State"-a practical method of grading the cognitive state of patients for the clinician. J Psychiatr Res 1975;12:189-98.

18 Tsai L, Tsuang MT. "Mini-Mental State" and computerized tomography. Am J Psychiatry 1979;136:436-9.

19 Reitan RM. An investigation of the validity of Halstead's measures of biological intelligence. Arch Neurol Psychiatry 1955;73:28-35.

20 Golden CJ. Validity of the Halstead-Reitan Neuropsychological Battery in a mixed psychiatric and brain injured population. $J$ Consult Clin Psychol 1977;45:1043-51.

21 Russell EW, Neuringer C, Goldstein G. Assessment of Brain Damage: a Neuropsychological Key Approach. New York: Wiley Interscience, 1970.
22 Lewis GP, Golden CJ, Moses JA Jr, Osmon DC, Purisch AD, Hammeke TA. Localization of cerebral dysfunction with a standardized version of Luria's neuropsychological battery. J Consult Clin Psychol 1978;47:1003-19.

23 Golden CJ, Hammeke TA, Purisch AD. Diagnostic validity of a standardized neuropsychological battery derived from Luria's neuropsychological tests. J Consult Clin Psychol 1978;46:1258-65.

24 Golden CJ. Item Interpretation of the Luria-Nebraska Neuropsychological Battery. Lincoln NE: University of Nebraska Press, 1982.

25 Gazzaniga MS, Bogen JE, Sperry RW. Observations of visual perception after disconnection of the cerebral hemisphere in man. Brain 1965;33:221-36.

26 Kimura D. Dual functional asymmetry of the brain in visual perception. Neuropsychologia 1966;4:275-85.

27 Kimura D. Spatial localization in left and right visual fields. Can J Psychol 1969;23:445-58.

28 Gazzaniga MS. The Bisected Brain. New York: Appleton-Century-Crofts, 1970.

29 Sperry RW. Lateral specialisation in the surgically separated hemispheres. In: Milner B, ed. Hemisphere Specialization and Interaction. Cambridge: The MIT Press, 1975:5-19.

30 Heilman KM, Valenstein E, eds. Clinical Neuropsychology. New York: Oxford University Press, 1979.

31 Mesulam MM. Principles of Behavioural Neurology. Philadelphia: FA Davis Co, 1985.

32 Kolb B, Whishew IQ. Fundamentals of Human Neuropsychology. San Francisco: WH Freeman and Co., 1980.

33 Fromm-Auch D. Neuropsychological assessment of depressed patients before and after drug therapy: clinical profile interpretation. In: Flor-Henry P, Gruzelier J, eds. Laterality and Psychopathology. Amsterdam: Elsevier Scientific Publishers, 1983:83-102.

34 Kronfol Z, Hamsher K deS, Digre K, Waziri R. Depression and hemispheric functions: changes associated with unilateral ECT. Br J Psychiatry 1978;132:560-7.

35 Taylor MA, Redfield J, Abrams R. Neuropsychological dysfunction in schizophrenia and affective disease. Biol Psychiatry 1981;16:467-78.

36 Donnelly EF, Dent JK, Murphy DL. Comparison of temporal lobe epileptics and affective disorders on the Halstead-Reitan test battery. $J$ Clin Psychol 1972;28:61-62.

37 Faber R, Abrams R, Taylor MA, Kasprisin A, Morris C, Weisz R. Comparison of schizophrenic patients with formal thought disorder and neurologically impaired patients with aphasia. Am $J$ Psychiatry 1983;140:1348-51.

38 Andreasen NC, Grove WM. The relationship between schizophrenic language, manic language and aphasia. In: Gruzelier J, Flor-Henry P, eds. Hemisphere Asymmetries of Function in Psychopathology. New York: Elsevier/North Holland, 1979:373-90.

39 Sackeim HA, Greenberg MS, Weiman AL, Gur RC, Hungerbuhler JP, Geschwind N. Hemisphere asymmetry in the expression of positive and negative emotions. Arch Neurol 1982;39:210-8. 